\title{
Anomalous Correlators in Nonlinear Dispersive Wave Systems
}

\author{
Joseph Zaleski®, ${ }^{1}$ Miguel Onorato $\odot,{ }^{2}$ and Yuri V. Lvov ${ }^{1, *}$ \\ ${ }^{1}$ Department of Mathematical Sciences, Rensselaer Polytechnic Institute, Troy, New York 12180, USA \\ ${ }^{2}$ Dipartimento di Fisica, Università di Torino and INFN, Sezione di Torino, \\ Via P. Giuria, 1-Torino, 10125, Italy
}

(Received 21 January 2020; revised manuscript received 21 March 2020; accepted 31 March 2020; published 26 May 2020)

\begin{abstract}
We show that Hamiltonian nonlinear dispersive wave systems with cubic nonlinearity and random initial data develop, during their evolution, anomalous correlators. These are responsible for the appearance of "ghost" excitations, i.e., those characterized by negative frequencies, in addition to the positive ones predicted by the linear dispersion relation. We use generalization of the Wick's decomposition and the wave turbulence theory to explain theoretically the existence of anomalous correlators. We test our theory on the celebrated $\beta$-Fermi-Pasta-Ulam-Tsingou chain and show that numerically measured values of the anomalous correlators agree, in the weakly nonlinear regime, with our analytical predictions. We also predict that similar phenomena will occur in other nonlinear systems dominated by nonlinear interactions, including surface gravity waves. Our results pave the road to study phase correlations in the Fourier space for weakly nonlinear dispersive wave systems.
\end{abstract}

DOI: 10.1103/PhysRevX.10.021043

Subject Areas: Nonlinear Dynamics, Statistical Physics

\section{INTRODUCTION}

Wave turbulence theory has led to successful predictions on the wave spectrum in many fields of physics [1,2]. In this framework the system is represented as a superposition of a large number of weakly interacting waves with the complex normal variables $a_{k}=a(k, t)$. In its essence, the classical wave turbulence theory is a perturbation expansion in the amplitude $a_{k}$ of the nonlinearity, yielding, at the leading order, to a system of quasilinear waves whose amplitudes are slowly modulated by resonant nonlinear interactions [1-6]. This modulation leads to a redistribution of the spectral energy density among length scales, and is described by a wave kinetic equation. One way to derive the wave kinetic equation is to use the random phase and amplitude approach developed in Refs. [2,7,8]. The initial state of the system can always be prepared so that the assumption of random phases and amplitudes is true. Whether the phases remain random in the evolution of the system has been an issue of intense discussions. In wave turbulence theory, the standard object to look at is the second-order correlator, $\left\langle a_{k}(t) a_{l}^{*}(t)\right\rangle$, where $\langle\cdots\rangle$ is an average over an ensemble of initial conditions with different random phases and amplitudes. As will be

\footnotetext{
* Corresponding author. lvovy@rpi.edu

Published by the American Physical Society under the terms of the Creative Commons Attribution 4.0 International license. Further distribution of this work must maintain attribution to the author(s) and the published article's title, journal citation, and DOI.
}

clear later on, under the homogeneity assumption, the second-order correlator is related to the wave action spectral density function, i.e., the wave spectrum, $n_{k}=n(k, t)$. However, one should note that the complex normal variable, as defined in the wave turbulence theory, is a complex function also in physical space. Therefore, the second-order statistics are not fully determined by the above correlator. The so-called "anomalous correlator," $\left\langle a_{k}(t) a_{l}(t)\right\rangle$, see Refs. $[9,10]$, needs also to be computed. Under the hypothesis of homogeneity, this will give the anomalous spectrum, $m_{k}=m(k, t)$, to be defined in the next section. Indeed, if phases are totally random, this quantity would be zero. We show that, in the nonlinear evolution of the system, this is not the case. Far from it, this quantity is strongly nonzero and, in the limit of weak nonlinearity, we predict analytically and verify numerically its value.

Our ideas are based on the extension of the wave turbulence theory to include these anomalous correlators. Notably, conventional wave turbulence theory has been successful in the understanding of the spectral energy transfer in complex wave systems such as the ocean [11], optics [12] and Bose-Einstein condensates [13], onedimensional chains [14], and magnets [15]. Analogously, anomalous correlators first appeared in the well-known Bardeen-Cooper-Schriffer theory of superconductivity [16]. Subsequently, anomalous correlators have been studied in $S$ theory $[9,10]$.

Recently, anomalous correlations were shown to play an important role in explaining numerical observations of nondecaying oscillations around a steady state in a turbulence-condensate system modeled by the nonlinear 
Schrödinger equation [17-19]. Such oscillations, corresponding to a fraction of the wave action being periodically converted from the condensate to the turbulent part of the spectrum, were shown to be directly due to phase coherence [17]. In Ref. [20], a system of coupled nonlinear Schrödinger equations has been considered and specific attention was focused on the phenomena of recurrence of incoherent waves observed in the early stages of the dynamics. The authors derived a variant of the kinetic equation which includes anomalous correlators; the peculiarity of such an equation is that it is capable of describing properly the recurrence phenomena observed in the simulations.

One of the main tools used to derive the theory is the Wick's contraction rule that allows one to split higherorder correlators as a sum of products of second-order correlators, plus cumulants. To explain analytically the existence of the anomalous correlators, it is necessary to use the more general form of the Wick's decomposition, namely the form that allows anomalous correlators. We then demonstrate that the anomalous correlators are responsible for creating the "ghost waves," i.e., the waves with the frequency equal to the negative of the frequency predicted by the linear dispersion relationship. These ideas are tested on a simple, but nontrivial, system, i.e., the $\beta$-Fermi-Pasta-Ulam-Tsingou (FPUT) chain. The chain model was introduced in the 1950s to study the thermal equipartition in crystals [21]: it consists of $N$ identical masses, each one connected by a nonlinear string; the elastic force can be expressed as a power series in the displacement from equilibrium. Fermi, Pasta, Ulam, and Tsingou integrated numerically the equations of motion and conjectured that, after many iterations, the system would exhibit a thermalization, i.e., a state in which the influence of the initial modes disappears and the system becomes random, with all modes excited equally (equipartition of energy) on average. Successful predictions on the timescale of equipartition have been recently obtained in Refs. [14,22,23] using the wave turbulence approach. In this paper, we perform extensive numerical simulations with initial random data and look at all the possible excitations, once a thermalized state has been reached. This is all done by analyzing the spatial-temporal $(k-\Omega)$ spectrum, i.e., the square of the space-time Fourier transform of the wave amplitudes. Analyses of the effective dispersion relation in the nonlinear system is a well-known and widely used theoretical and numerical tool; see, e.g., Ref. [24].

We give numerical evidence that in addition to the "normal" waves with frequency $\omega$ predicted by the linear dispersion relation for wave number $k$, there are the "ghost" excitations with the negative frequencies. Our theoretical analysis reveals that the origin of those ghost excitations resides on the nonzero values of the second-order anomalous correlator.

\section{MODEL}

The theory that we develop hereafter applies to any system with cubic nonlinearity. Examples of such systems, among others, include deep water surface gravity waves [25], nonlinear Klein-Gordon [22], and the $\beta$-FermiPasta-Ulam-Tsingou chain. In normal variables $a_{k}$ the Hamiltonian of these systems assumes the canonical form:

$$
\begin{aligned}
H= & \sum_{k} \omega_{k}\left|a_{k}\right|^{2}+\sum_{k_{1}, k_{2}, k_{3}, k_{4}}\left[\left(T_{1234}^{(1)} a_{1}^{*} a_{2} a_{3} a_{4}+\text { c.c. }\right) \delta_{1}^{234}\right. \\
& +\frac{1}{2} T_{1234}^{(2)} a_{1}^{*} a_{2}^{*} a_{3} a_{4} \delta_{12}^{34} \\
& \left.+\frac{1}{4} T_{1234}^{(4)}\left(a_{1}^{*} a_{2}^{*} a_{3}^{*} a_{4}^{*}+\text { c.c. }\right) \delta_{1234}\right]
\end{aligned}
$$

where $\omega_{k}=\omega(k)$ are the positive frequencies associated to the wave numbers via the dispersion relation, $T_{1234}^{(i)}$ are coefficients that depend on the problem considered and satisfy specific symmetries for the system to be Hamiltonian, c.c. implies complex conjugation, $a_{j}=$ $a\left(k_{j}, t\right)$ are the complex normal variables, and $\delta_{i j}^{l m}=\delta\left(k_{i}+\right.$ $\left.k_{j}-k_{l}-k_{m}\right)$ is the Kronecker delta. We assume that the only resonant interactions possible are the ones for which the following two relations are satisfied for a set of wave numbers:

$k_{1}+k_{2}=k_{3}+k_{4}, \quad \omega\left(k_{1}\right)+\omega\left(k_{2}\right)=\omega\left(k_{3}\right)+\omega\left(k_{4}\right)$.

With the objective of presenting some comparison with numerical simulations, out of many physical systems described by the above Hamiltonian, we select a simple one-dimensional system, the $\beta$-Fermi-Pasta-Ulam-Tsingou chain. Modeling a vibrating string, this problem consists of a system of $N$ identical particles connected locally to each other by a nonlinear oscillator. In physical space in terms of the displacements with respect to the equilibrium position $q_{j}(t)$ and their momenta $p_{j}(t)$, the Hamiltonian takes the following form:

$$
H=H_{2}+H_{4},
$$

with

$$
\begin{aligned}
& H_{2}=\sum_{j=1}^{N}\left(\frac{1}{2} p_{j}^{2}+\frac{1}{2}\left(q_{j}-q_{j+1}\right)^{2}\right), \\
& H_{4}=\frac{\beta}{4} \sum_{j=1}^{N}\left(q_{j}-q_{j+1}\right)^{4} .
\end{aligned}
$$

$\beta$ is the nonlinear spring coefficient (without loss of generality, we have set the masses and the linear spring 
constant equal to 1). Newton's law in physical space is given by

$\ddot{q}_{j}=\left(q_{j+1}+q_{j-1}-2 q_{j}\right)+\beta\left[\left(q_{j+1}-q_{j}\right)^{3}-\left(q_{j}-q_{j-1}\right)^{3}\right]$.

We assume periodic boundary condition; our approach is developed in Fourier space and the following definitions of the direct and inverse discrete Fourier transforms are adopted:

$Q_{k}=\frac{1}{N} \sum_{j=0}^{N-1} q_{j} e^{-i 2 \pi k j / N}, \quad q_{j}=\sum_{k=-N / 2+1}^{N / 2} Q_{k} e^{i 2 \pi j k / N}$,

where $k$ are discrete wave numbers and $Q_{k}$ are the Fourier amplitudes. The displacement $q_{j}$ and momentum $p_{j}$ of the $j$ particle are linked by canonically conjugated Hamilton equations:

$$
\dot{p}_{j}=-\frac{\partial H}{\partial q_{j}}, \quad \dot{q}_{j}=\frac{\partial H}{\partial p_{j}} .
$$

We then perform the Fourier transformation to Fourier images of position and momenta, and then additional canonical transformation to complex amplitude $a_{k}$ given by

$$
a_{k}=\frac{1}{\sqrt{2 \omega_{k}}}\left(\omega_{k} Q_{k}+i P_{k}\right),
$$

where $\omega_{k}=2|\sin (\pi k / N)|>0$ and $Q_{k}$ and $P_{k}$ are the Fourier amplitudes of $q_{j}$ and $p_{j}$, respectively. In terms of $a_{k}$ the equation of motion reads, see Ref. [26],

$$
\begin{aligned}
i \frac{d a_{1}}{d t}= & \omega_{k_{1}} a_{1}+\sum_{k_{2}, k_{3}, k_{4}}\left(T_{1234}^{(1)} a_{2} a_{3} a_{4} \delta_{1}^{234}+T_{1234}^{(2)} a_{2}^{*} a_{3} a_{4} \delta_{12}^{34}\right. \\
& \left.+T_{1234}^{(3)} a_{2}^{*} a_{3}^{*} a_{4} \delta_{123}^{4}+T_{1234}^{(4)} a_{2}^{*} a_{3}^{*} a_{4}^{*} \delta_{1234}\right)
\end{aligned}
$$

where all wave numbers $k_{2}, k_{3}$, and $k_{4}$ are summed from 0 to $N-1$ and $\delta_{a b . .}^{c d .}=\delta\left(k_{a}+k_{b}+\cdots-k_{c}-k_{d}-\cdots\right)$ is the generalized Kronecker delta that accounts for a periodic Fourier space; i.e., its value is one when the argument is equal to $0(\bmod N)$. The matrix elements $T_{1234}^{(1)}, T_{1234}^{(2)}, T_{1234}^{(3)}$ prescribe the strength interactions of wave numbers $k_{1}, k_{2}$, $k_{3}$, and $k_{4}$. Their values are given in the Appendix A.

\section{A. $(k-\Omega)$ spectrum}

The main statistical object discussed in this paper is the wave number-frequency $(k-\Omega)$ spectrum. Starting from the complex amplitude $a(k, t)$ we take the Fourier transform in time so that we get $a(k, \Omega)$; under the hypothesis of homogeneous and stationary conditions, the second-order $(k-\Omega)$ correlator takes the following form,

$\left\langle a\left(k_{i}, \Omega_{p}\right) a\left(k_{j}, \Omega_{q}\right)^{*}\right\rangle=N\left(k_{i}, \Omega_{p}\right) \delta\left(k_{i}-k_{j}\right) \delta\left(\Omega_{p}-\Omega_{q}\right)$,

where $\langle\cdots\rangle$ implies averages over initial conditions with different random phases. $N(k, \Omega)$ is the $(k-\Omega)$ spectrum defined as follows:

$N^{(a)}(k, \Omega)=\frac{1}{2 \pi} \frac{1}{N} \int_{-\infty}^{+\infty} \sum_{l=1}^{N} R(l, \tau) e^{-i 2 \pi k l / N} e^{-i \Omega \tau} d \tau$,

where $R(l, \tau)=\left\langle a_{j}(t)^{*} a_{j+l}(t+\tau)\right\rangle$ is the space-time autocorrelation function.

Linear $(k-\Omega)$ spectrum.-Before diving into the nonlinear dynamics, we discuss the predictions in the linear regime. Therefore, we start by neglecting the nonlinearity in Eq. (8) and find the solution in the form

$$
a_{k}(t)=a_{k}\left(t_{0}\right) e^{-i \omega_{k} t},
$$

where $t_{0}$ is a time at which the solution is known or an initial condition. We then take the Fourier transform in time:

$$
a(k, \Omega)=a\left(k, t_{0}\right) \delta\left(\Omega-\omega_{k}\right) .
$$

After multiplication by its complex conjugate and taking averages over different realizations with the same statistics, we get

$$
N^{(a)}(k, \Omega)=n^{(a)}\left(k, t_{0}\right) \delta\left(\Omega-\omega_{k}\right),
$$

where $n^{(a)}\left(k, t_{0}\right)$ is the standard wave spectrum at time $t_{0}$ related to the second-order correlator as

$$
\left\langle a\left(k_{i}, t_{0}\right) a\left(k_{i}, t_{0}\right)^{*}\right\rangle=n^{(a)}\left(k_{i}, t_{0}\right) \delta\left(k_{i}-k_{j}\right),
$$

and defined via the autocorrelation function as

$$
n^{(a)}\left(k_{i}, t_{0}\right)=\frac{1}{N} \sum_{l}\left\langle a_{j}\left(t_{0}\right) a_{j+l}\left(t_{0}\right)^{*}\right\rangle e^{-i 2 \pi k l / N} .
$$

In the linear regime $n^{(a)}\left(k_{i}, t_{0}\right)$ does not evolve in time.

Equation (13) implies that in the linear case the $(k-\Omega)$ spectrum is different from zero only for those values of $\Omega$ and $k$ for which the dispersion relation is satisfied. Note that in this formulation $\omega_{k}$ is defined as a positive quantity; therefore, only the positive branch of the dispersion relation curve appears in the linear regime. 

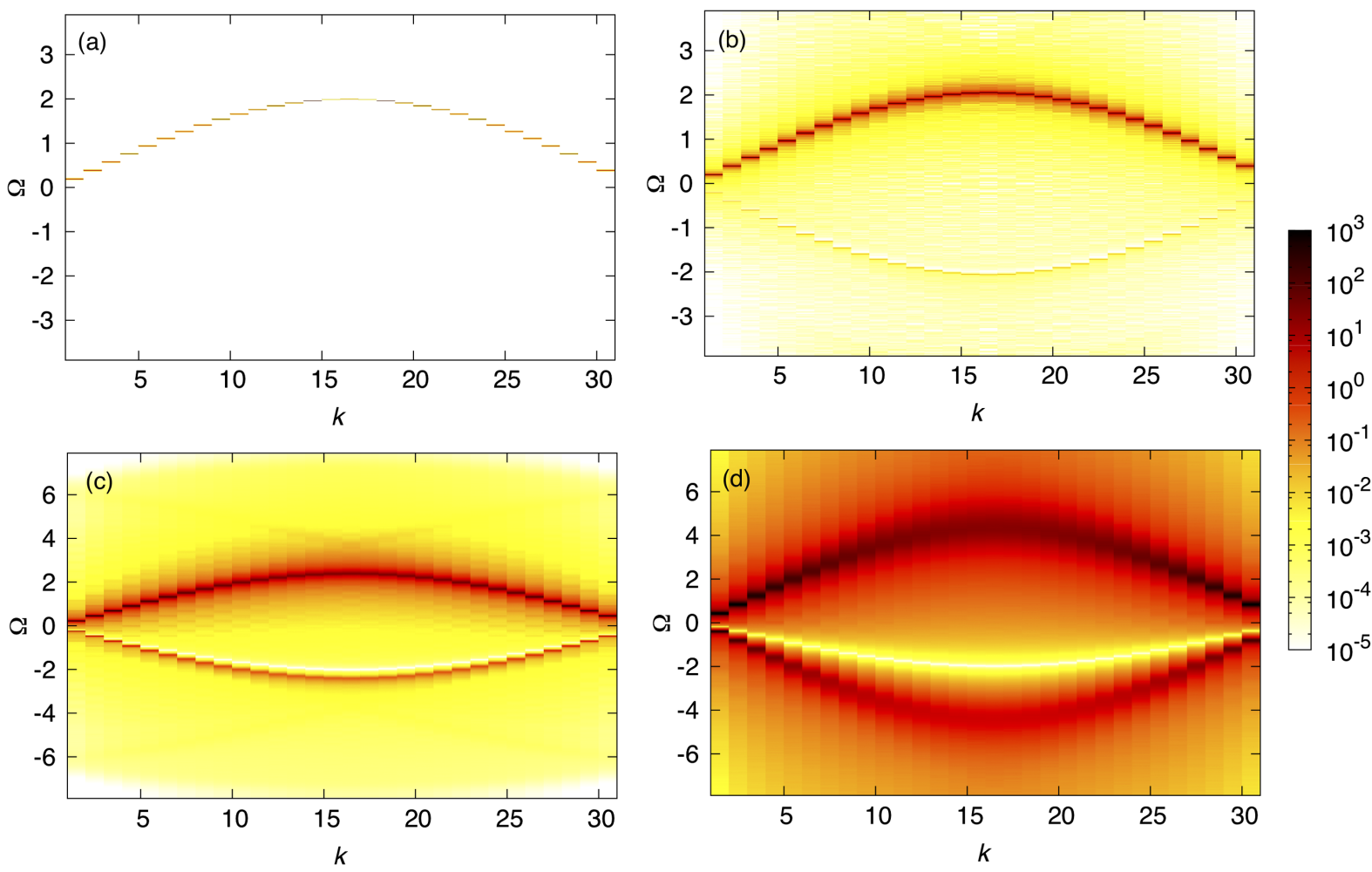

FIG. 1. $(k-\Omega)$ spectrum, $N^{(a)}(k, \Omega)$, for different values of $\epsilon$ : (a) $\epsilon=0$, (b) $\epsilon=0.0089$ (c) $\epsilon=0.089$ (d) $\epsilon=1.12$. In the linear case, (a), the $N^{(a)}(k, \Omega)$ is different from 0 only when the frequency $\Omega$ matches the linear dispersion relation. As the nonlinearity is increased, (b)-(d), a frequency shift, a broadening of the frequencies, and a lower branch less intense than the upper one are visible. Waves with negative frequencies are named "ghost" excitations.

\section{B. Numerical results for the $(k-\Omega)$ spectrum}

We now test the predictions from Eq. (13) in both the linear and nonlinear regimes. We perform numerical simulations of Eq. (5) using a symplectic algorithm; see Ref. [27]. We use 32 particles in the simulations; such a choice is completely uninfluential for the results presented below. In the linear regime, we just prescribe a thermalized spectrum with some initial random phases of the wave amplitudes $a_{k}$ and evolve the system in time up to a desired final time; a Fourier transform in time is then taken to build the $(k-\Omega)$ spectrum. In the nonlinear regime we perform long simulations up to a thermalized spectrum. For a given nonlinearity, 1000 realizations characterized by different random phases are made and ensemble averages are considered to compute the $(k-\Omega)$ spectrum. All simulations have the same initial linear energy and, from an operative point of view, the only difference between them is the value of $\beta$. To characterize the strength of the nonlinearity, we use the following ratio between nonlinear and linear Hamiltonians at the beginning of each simulation:

$$
\epsilon=\frac{H_{4}}{H_{2}} \propto \beta .
$$

Results are shown in Fig. 1, where, for different values of the nonlinear parameter $\epsilon$, the spectrum $N^{(a)}(k, \Omega)$ is plotted using a colored logarithmic scale. We first focus our attention on the linear regime, $\epsilon=0$. Results are shown in Fig. 1(a). As well predicted by the theory, the plot shows dots in the positive frequency plane, where the frequencies $\Omega$ and wave numbers $k$ satisfy the linear dispersion curve $\omega_{k}$. Increasing the nonlinearity, Figs. 1(b)-1(d), two wellknown effects appear. The first one is a shift of the frequencies, due to nonlinearity [this is more evident in Figs. 1(c) and 1(d) where the frequency scale in the vertical axes has been changed]. The second one is the broadening of the frequencies. This is related to the fact that the amplitude for each wave number is not constant in time; therefore, the amplitude-dependent frequencies are not constant in time and they oscillate around a mean value with some fluctuations. Those results are well understood, at least in the weakly nonlinear regime, and can be predicted using wave turbulence tools; see Refs. [2,23]. Besides these two effects, starting from Fig. 1(b), the presence of a lower branch, whose intensity is much less than the upper one, starts to be visible. The lower curve becomes more important and, when the nonlinearity is of order one, is of the same order of magnitude as the upper one. The total number of waves in the simulation $N_{\text {tot }}$ is given by the integral over $\Omega$ and the sum over all $k$ of the function $N^{(a)}(k, \Omega)$. In the weakly nonlinear regime, $N_{\text {tot }}$ is an adiabatic invariant of the equation of motion (5); the 


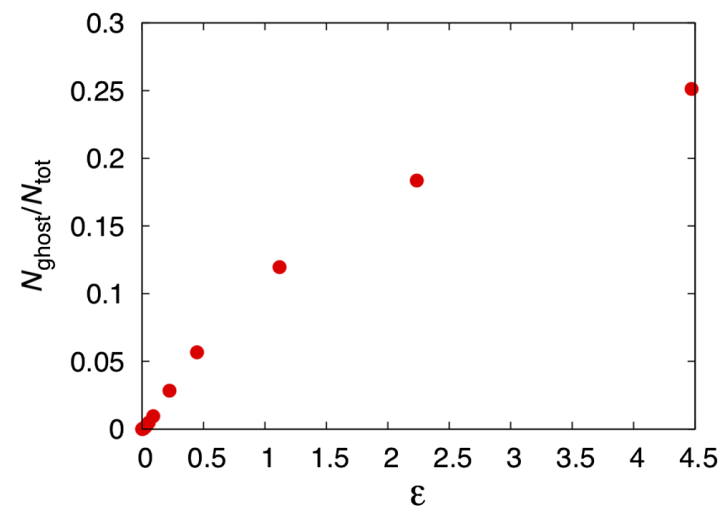

FIG. 2. Ratio between the number of ghost excitations $N_{\text {ghost }}$ over the total number of waves $N_{\text {tot }}$ as a function of the nonlinearity.

plot highlights the existence of waves with negative frequencies, which will be named "ghost" excitations. One of the scopes of the present paper is the understanding of the origin of such waves. Before entering into the discussion, we show in Fig. 2 the ratio of ghost excitations $N_{\text {ghost }}$, i.e., $N^{(a)}(k, \Omega)$ integrated over negative frequencies and summed over all wave numbers, divided by the total number of waves $N_{\text {tot }}$. As can be seen from the plot, there is a monotonic growth of the ghost waves that, for very large nonlinearity, can reach values up to $25 \%$ of the total number.

\section{ANOMALOUS CORRELATORS}

To explain the presence of ghost excitations, we introduce the so-called second-order anomalous correlator $[9,10,20]$ :

$$
\left\langle a_{k}(t) a_{j}(t)\right\rangle=m_{k}(t) \delta(k+j),
$$

with the anomalous spectrum defined as

$$
m_{k}^{(a)}(t)=\frac{1}{N} \sum_{l}\left\langle a_{j} a_{j+l}\right\rangle e^{-i 2 \pi k l / N} .
$$

Similarly, we also introduce the second-order $(k-\Omega)$ anomalous correlator:

$$
\left\langle a\left(k_{i}, \Omega_{l}\right) a\left(k_{j}, \Omega_{m}\right)\right\rangle=M^{(a)}\left(k_{i}, \Omega_{l}\right) \delta\left(k_{i}+k_{j}\right) \delta\left(\Omega_{l}+\Omega_{m}\right),
$$

where

$$
M^{(a)}(k, \Omega)=\frac{1}{2 \pi} \frac{1}{N} \int_{-\infty}^{+\infty} \sum_{l=1}^{N} S(l, \tau) e^{-i 2 \pi k l / N} e^{-i \Omega \tau} d \tau
$$

and $S(l, \tau)=\left\langle a_{j}(t) a_{j+l}(t+\tau)\right\rangle$. The presence in Eqs. (17) and (19) of the Kronecker $\delta$ over wave numbers and the Dirac $\delta$ over frequency are related to the hypothesis of statistical homogeneity and stationarity, respectively. Note that $M^{(a)}(k, \Omega)$ is not the Fourier transform in time of $m_{k}^{(a)}(t)$ and, in general, both can be complex functions. To verify numerically that the anomalous correlator is indeed nonzero, we measure numerically the real part of the second-order correlator $\left\langle a_{k_{i}}(t) a_{k_{j}}(t)\right\rangle$ as a function of $k_{1}$ and $k_{2}$. Results are plotted in Fig. 3 where we show the results of two numerical simulations characterized by two different values of the nonlinear parameter: Fig. 3(a) $\epsilon=$ 0.0089 and Fig. 3(b) $\epsilon=1.12$. In both cases, a diagonal contribution is visible, pointing out the existence of anomalous correlators in the $\beta$-FPUT model.

Generalization of the Wick's decomposition.-Using Eq. (17), it is straightforward to extend the Wick's decomposition by taking into account the anomalous correlators, as done in Ref. [15]:

$$
\begin{aligned}
& \left\langle a_{k}^{*} a_{l}^{*} a_{p} a_{n}\right\rangle=n_{k} n_{l}\left(\delta_{p}^{k} \delta_{n}^{l}+\delta_{n}^{k} \delta_{p}^{l}\right)+m_{k}^{*} m_{p} \delta_{k l} \delta_{p n}, \\
& \left\langle a_{k}^{*} a_{l} a_{p} a_{n}\right\rangle=n_{k} m_{p}\left(\delta_{k}^{l} \delta_{p n}+\delta_{k}^{n} \delta_{l p}\right)+n_{k} \delta_{k}^{p} m_{l} \delta_{n l}, \\
& \left\langle a_{k} a_{l} a_{p} a_{n}\right\rangle=m_{k} m_{l}\left(\delta_{k p} \delta_{l n}+\delta_{k l} \delta_{p n}+\delta_{k l} \delta_{p n}\right) .
\end{aligned}
$$
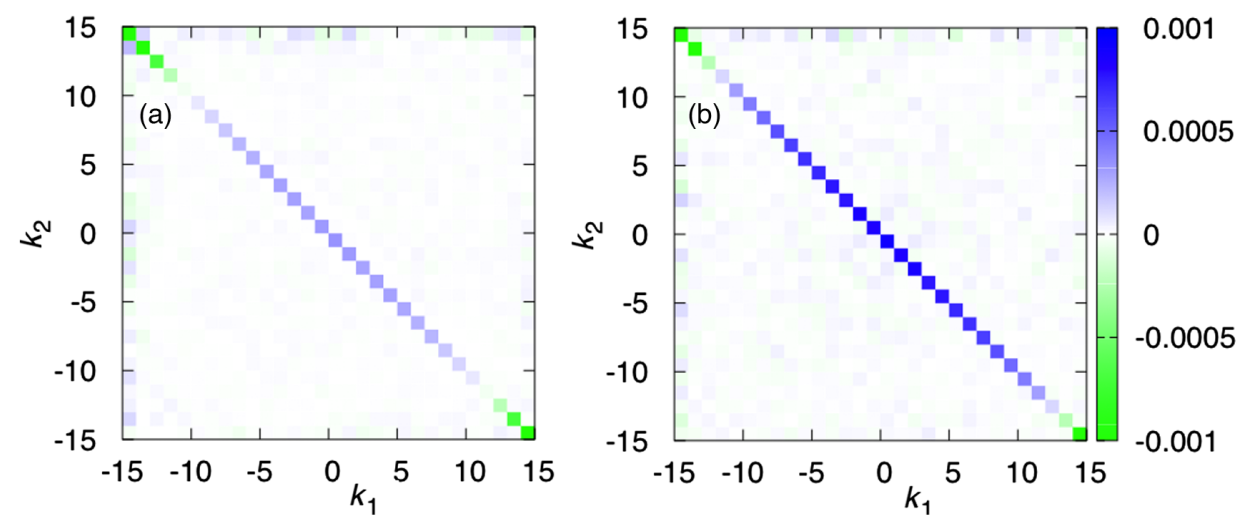

FIG. 3. The real part of the second-order anomalous correlator, $\operatorname{Re}\left[\left\langle a_{k_{1}} a_{k_{2}}\right\rangle\right]$, for (a) $\epsilon=0.0089$, (b) $\epsilon=1.12$. A diagonal contribution corresponding to $k_{2}=-k_{1}$ is evident in both panels. As the nonlinearity is increased, the contribution becomes larger. 

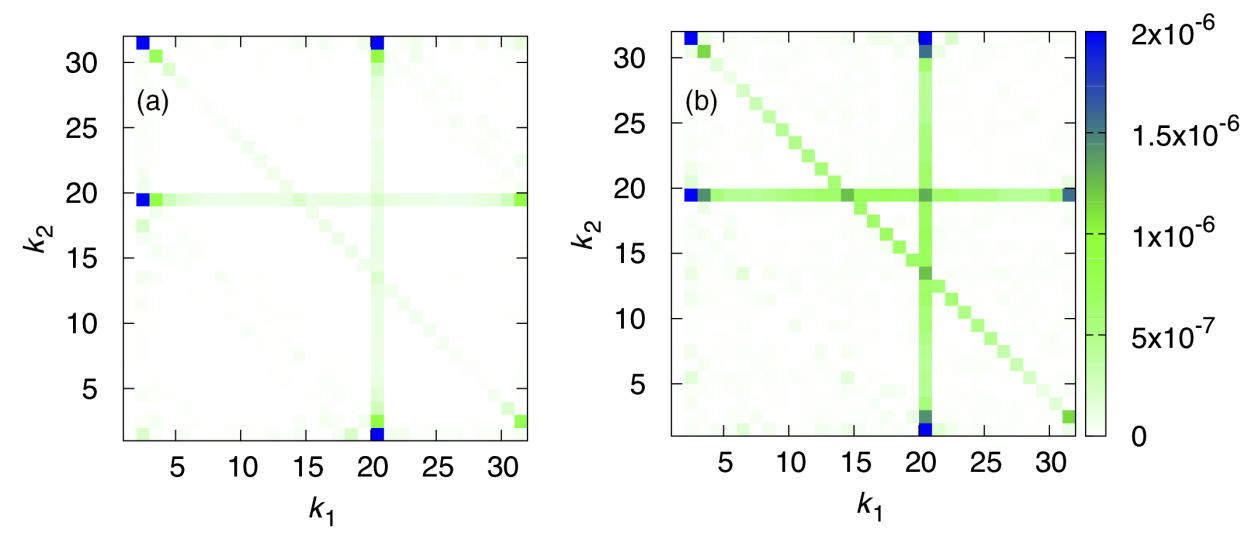

FIG. 4. Fourth-order correlator $\left|\operatorname{Re}\left[\left\langle a_{k_{1}} a_{k_{2}} a_{k_{3}}^{*} a_{k_{1}+k_{2}-k_{3}}^{*}\right\rangle\right]\right|$ with $k_{3}=20$. (a) $\epsilon=0.0089$, (b) $\epsilon=1.12$. Different horizontal, vertical, and diagonal lines are visible. Horizontal and vertical lines correspond to trivial resonances: $k_{1}=k_{3}$, vertical line; $k_{2}=k_{3}$, horizontal line; $k_{2}=-k_{1}+N$, diagonal line. The latter line corresponds to the presence of an anomalous second-order correlator. The intensity of the lines is larger for larger nonlinearity.

The above relations will be fundamental for making a natural closure of the moments when calculating analytically the $(k-\Omega)$ spectrum.

In Fig. 4, we find further evidence justifying this decomposition by plotting the real part of the fourth-order correlator $\left\langle a_{k_{1}} a_{k_{2}} a_{k_{3}}^{*} a_{k_{1}+k_{2}-k_{3}}^{*}\right\rangle$ with $k_{3}=20$, computed from numerical simulations for Fig. 4(a) $\epsilon=0.0089$ and Fig. 4(b) $\epsilon=1.12$. The diagonal lines in both figures, highlighting the contribution from the second-order anomalous correlator, are noticeable. The vertical and horizontal lines correspond to the trivial resonances in which two wave numbers are equal $(\bmod N)$.

\section{A. Theoretical prediction for the anomalous correlator in the weakly nonlinear regime}

A key step for the development of a theory for the anomalous correlator is the change of variable (near identity transformation) which allows one to remove bound modes, i.e., those modes that are phase locked to free modes and do not obey the linear dispersion relation. The procedure is well known in Hamiltonian mechanics and well documented, e.g., in Ref. [1]. We accomplish this via the following canonical transformation from variable $a_{k}(t)$ to $b_{k}(t)$ :

$$
\begin{aligned}
a_{1}= & b_{1}+\sum_{k_{2}, k_{3}, k_{4}}\left[B_{1234}^{(1)} b_{2} b_{3} b_{4} \delta_{1}^{234}+B_{1234}^{(3)} b_{2}^{*} b_{3}^{*} b_{4} \delta_{123}^{4}\right. \\
& \left.+B_{1234}^{(4)} b_{2}^{*} b_{3}^{*} b_{4}^{*} \delta_{1234}\right],
\end{aligned}
$$

with the coefficients $B_{1234}^{(i)}$ selected in such a way as to remove nonresonant terms in the original Hamiltonian [28]. Their values are given in Appendix A.

The transformation is asymptotic in the sense that the small amplitude approximation is made and the terms in the sums on the right-hand side are much smaller than the leading-order term $b_{1}$. The evolution equation for variable $b_{k}(t)$ contains resonant interactions and takes the following standard form:

$i \frac{d b_{1}}{\partial t}=\omega_{1} b_{1}+\sum_{k_{2}, k_{3}, k_{4}} T_{1234}^{(2)} b_{2}^{*} b_{3} b_{4} \delta_{12}^{34}+$ higher-order terms

where higher-order terms arising from the transformation have been neglected.

Using the transformation (22) and the generalized Wick's decomposition (21), we can now build the time-averaged anomalous spectrum (for details, see Appendix B):

$$
\left\langle m_{k}^{(a)}(t)\right\rangle_{t}=2\left(n_{k}^{(a)}+n_{-k}^{(a)}\right) \sum_{j} B_{k,-k, j, j}^{(3)} n_{j}^{(a)},
$$

where $\langle\cdots\rangle_{t}$ implies averaging over time. For the $\beta$-FPUT system in thermal equilibrium, where $n_{k}^{(a)}=T / \omega_{k}$ with $T$ constant, Eq. (23) reduces to

$$
\omega_{k}\left|\left\langle m_{k}^{(a)}(t)\right\rangle_{t}\right|=\frac{3 N T^{2} \beta}{2} .
$$

In Fig. 5, we compare this prediction for $\omega_{k}\left|\left\langle m_{k}^{(a)}(t)\right\rangle_{t}\right|$ in thermal equilibrium to the values given by numerical simulations for varying values of nonlinearity: the results are in good agreement in the weakly nonlinear regime, $\epsilon<0.1$. Here 500 ensembles were used to build the correlator $m_{k}(t)$; the subsequent time-averaging window used was $10^{5}$ with a sample spacing of $\Delta t=0.1$. For larger nonlinearity, it is expected that higher-order terms play a role in the evolution of the anomalous correlator.

In Fig. 6, we show the time evolution of the first five modes of $\left|\left\langle\omega_{k} m_{k}^{(a)}\left(t_{a}\right)\right\rangle_{t_{a}<t}\right|$, where ensemble averaging is used to build $m_{k}^{(a)}(t)$ and time averaging is used over the window $0<t_{a}<t$ to remove fast oscillations 


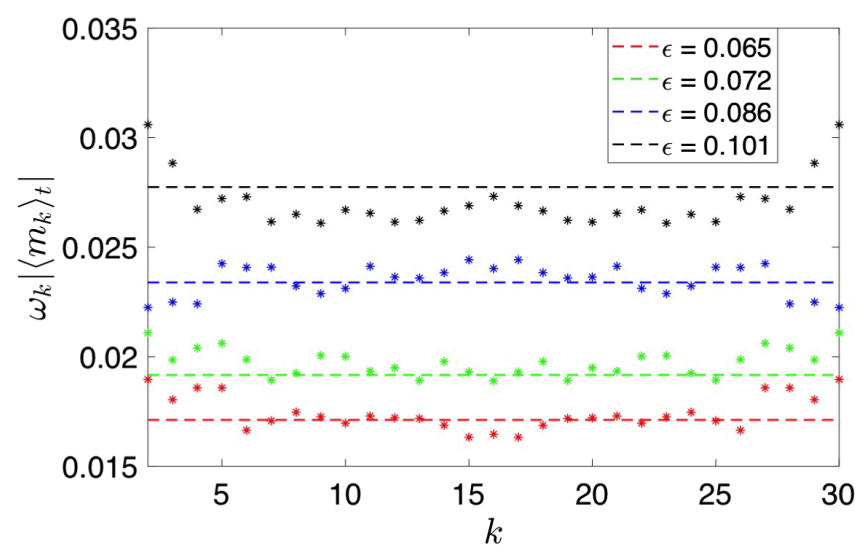

FIG. 5. Comparison of anomalous spectrum $\omega_{k}\left|\left\langle m_{k}^{(a)}(t)\right\rangle_{t}\right|$ as observed in numerical simulations (dots) with theoretical predictions given by Eq. (24) (dashed lines) for $\epsilon=0.065,0.072$, 0.086 , and 0.101 .

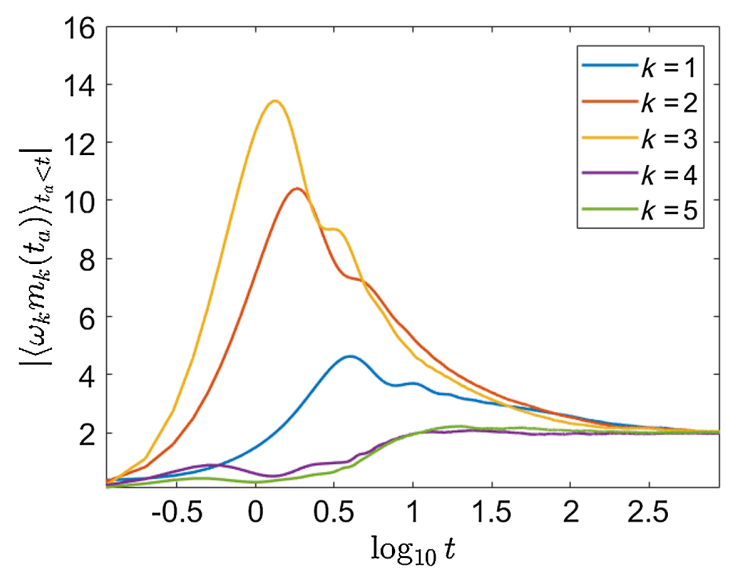

FIG. 6. Time evolution of the first five modes of the averaged quantity $\left|\left\langle\omega_{k} m_{k}^{(a)}\left(t_{a}\right)\right\rangle_{t_{a}<t}\right|$ from $t=0$ to $t=1000$, with $\epsilon=10$.

[see Eq. (B5)]. We verify that this quantity is indeed initially zero due to the randomness of phases. Here we use a larger value of nonlinearity $\epsilon=10$ to show the development of the anomalous correlator in a shorter time window. The amplitudes were initialized so that $\mid a_{k}(t=$ $0) \mid=\sqrt{[(N-k) / 2]}$ for $k= \pm 1, \pm 2, \pm 3$, with higher modes zero. The phases were initially normally distributed. We observe that the anomalous correlator grows with time, reaching a peak in modes 1,2 , and 3, before it eventually saturates between all modes equally, with $\omega_{k}\left|\left\langle m_{k}^{(a)}\left(t_{a}\right)\right\rangle_{t<t_{a}}\right|$ being constant for large times, as expected from our prediction Eq. (23).

\section{THEORETICAL PREDICTION FOR GHOST EXCITATIONS}

We have now developed all the tools for predicting analytically the $(k-\Omega)$ spectrum as defined in Eq. (9). Taking the Fourier transform in time of the canonical

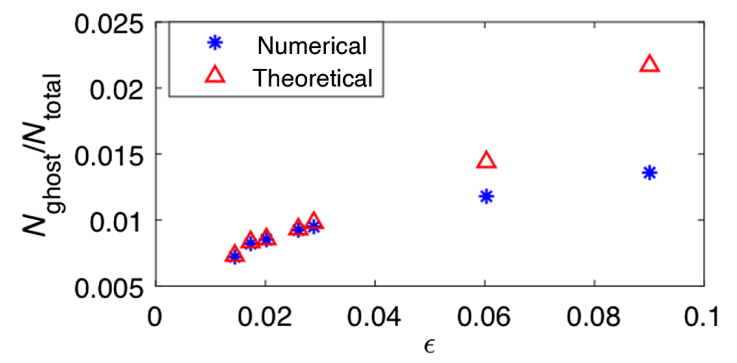

FIG. 7. The ratio between the number of ghost excitations $N_{\text {ghost }}$ over the total number of waves $N_{\text {tot }}$ as a function of the nonlinearity. Values given by numerical simulations (blue) are compared with the theoretical predictions (red) given by Eq. (27).

transformation (see Appendix C), using the generalized Wick's decomposition and the hypothesis of statistical stationarity and homogeneity, we get at leading order:

$$
\begin{aligned}
N^{(a)}(k, \Omega)= & n^{(b)}\left(k, t_{0}\right) \delta\left(\Omega-\omega_{k}\right) \\
& +F(k) \operatorname{Re}\left[m^{(b)}\left(k, t_{0}\right)\right] \delta\left(\Omega+\omega_{k}\right),
\end{aligned}
$$

with

$$
F(k)=4 \int \sum_{l} B_{k l k l}^{(3)} N^{(b)}\left(l, \Omega_{p}\right) d \Omega_{p}
$$

where we have used the fact that at the leading order, $m^{(b)}(k, t) \simeq m^{(a)}(k, t)$ and $n^{(b)}\left(k, t_{0}\right) \simeq n^{(a)}\left(k, t_{0}\right)$. Equation (25) predicts the presence of the upper and lower branch in the $(k-\Omega)$ plane. The presence of ghost excitations is clearly related to the second-order anomalous correlator. We can now predict the percentage of ghost excitations as

$$
\frac{N_{\text {ghost }}}{N_{\text {tot }}}=\frac{\sum_{k} \operatorname{Re}\left[m^{(a)}\left(k, t_{0}\right)\right] F(k)}{\sum_{k}\left\{n^{(a)}\left(k, t_{0}\right)+\operatorname{Re}\left[m^{(a)}\left(k, t_{0}\right)\right] F(k)\right\}} .
$$

In Fig. 7, we plot the ratio as determined by Eq. (27) compared with the ratio observed in our simulations for several values of nonlinearity. We find that the results agree for small values of nonlinearity $\epsilon<0.03$; for larger nonlinearity, the theoretical prediction is considerably larger.

\section{NONLINEAR STANDING WAVES}

The development of a regime characterized by an anomalous spectrum corresponds to a tendency for the system to develop standing waves in the original displacement variable $q_{j}(t)$. Indeed, the existence of an anomalous spectrum implies a correlation between positive and negative wave numbers. The connection between the anomalous correlator and standing waves can be seen in the following illustrative example. Consider the restrictive ensemble of realizations of the linear system where amplitudes and phases are initiated in Fourier space with 
a correlation between wave numbers $k=1$ and $k=-1$. Namely, let us initialize the system with equal amplitudes and opposing phases:

$$
a_{k}(t=0)= \begin{cases}A_{1} e^{-i \phi_{1}} & \text { if } k=1 \\ A_{1} e^{i \phi_{1}} & \text { if } k=-1 \\ 0 & \text { otherwise }\end{cases}
$$

with the random phase $\phi_{1}$. In terms of the displacement variables, this would correspond to the system being initially at rest and displaced from equilibrium as a single wave:

$$
q_{j}(t=0)=2 A_{1} \sqrt{\frac{2}{\omega_{1}}} \cos \left(\frac{2 \pi j}{N}-\phi_{1}\right) .
$$

Since the system is assumed to be linear, the time evolution of complex amplitudes $a_{1}$ and $a_{-1}$ will be given by

$$
a_{ \pm 1}(t) \simeq A_{1} e^{-i\left(\omega_{1} t \pm \phi_{i}\right)}
$$

Averaging over random phase $\phi_{1}$, the anomalous correlator becomes

$$
m_{1}=\left\langle a_{1} a_{-1}\right\rangle=A_{1}^{2} e^{-2 i \omega_{1} t},
$$

analogous to the oscillating term of Eq. (B5) for the anomalous correlator in the nonlinear case with amplitudes and phases being initially completely random. In terms of displacement, such initial conditions give

$$
q_{j}(t)=2 A_{1} \sqrt{\frac{2}{\omega_{1}}} \cos \left(\frac{2 \pi j}{N}-\phi_{1}\right) \cos \left(\omega_{1} t\right),
$$

which corresponds to the standing wave pattern. Thus, we see that the phase and amplitude correlations which result in a nonzero anomalous correlator are directly linked to the formation of standing waves in this particular example.

This consideration can be generalized for the case of weakly nonlinear systems and more general initial conditions. Indeed, for weakly nonlinear systems the amplitudes $\left|a_{1}\right|$ and $\left|a_{-1}\right|$ will be changing slowly over many oscillations, thus maintaining strongly nonzero anomalous correlations and standing waves.

In Fig. 8(a), we numerically solve the equations of motion with initial conditions given by Eq. (28). Here we plot a color map of the displacement $q_{j}(t)$ for all masses as a function of time as the system reaches the timescale required for statistical thermal equilibrium. The nonlinearity parameter $\epsilon=4.74$, in the regime of strong nonlinearity and outside the regime of validity of our theory. Nevertheless, we initially consider this example to display how the system behaves when the phase correlations develop rapidly. The existence of several regions of
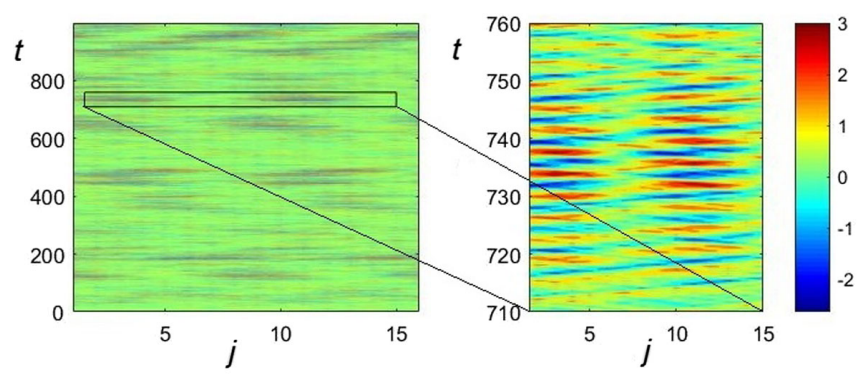

FIG. 8. Color map of the displacement $q_{j}(t)$ for the system with $\epsilon=4.74$ initialized with particles at rest with initial positions as a single sine wave. A nonlinear standing wave pattern is visible.

standing wave behavior are clearly visible as darker regions in the image, as the inset of Fig. 8(b) shows.

It is important to emphasize that Fig. 8 shows a single realization of the system, while correlators $m_{k}(t), n_{k}(t)$ describe statistical ensemble-averaged quantities. Thus the existence of the standing wave patterns is not in violation of the presumed assumption of spatial homogeneity.

Below we give numerical evidence that such coherent structures can also be observed for smaller values of nonlinearity that are within the regime of validity of our theory.

In Fig. 9(a), we plot the displacement as a function of time for the system with $\epsilon=0.02$, a value of nonlinearity well within the regime of agreement of our theory, as shown in Figs. 7 and 5. Here, we prescribe initial conditions so that the total energy is initially in the first wave number, i.e., $a_{k}(t=0)=0$ for all $k \neq 1$, and plot a single realization. This corresponds to a pure traveling wave solution in the linear system; indeed, as seen in Fig. 9(a), the system is initially a traveling wave, represented by series of slanted parallel lines in the color map of $q_{j}(t)$. Conversely, in Fig. 9(b), we show that by the time the system has reached the timescale required for statistical thermal equilibrium, a prominent standing wave has developed, due to the phase correlations between positive and negative lowest wave numbers. Notably, phase correlations are not restricted to only the lowest wave numbers. To emphasize this, we consider the following spatial frequency filter applied to the displacement,

$$
\tilde{q}_{j}(t)=\sum_{k=-N / 2+1}^{N / 2} H_{k} Q_{k} e^{i 2 \pi j k / N},
$$

where

$$
H_{k}= \begin{cases}1 & \text { if } k=5,6 \\ 0 & \text { otherwise }\end{cases}
$$

is selected to only show the waves with frequencies corresponding to $k=5,6$.

We plot the resulting color map of $\tilde{q}_{j}(t)$ in Fig. 9(c), with Fig. 9(d) showing a closer look at the boxed region in Fig. 9(c). Here we clearly still observe these standing 

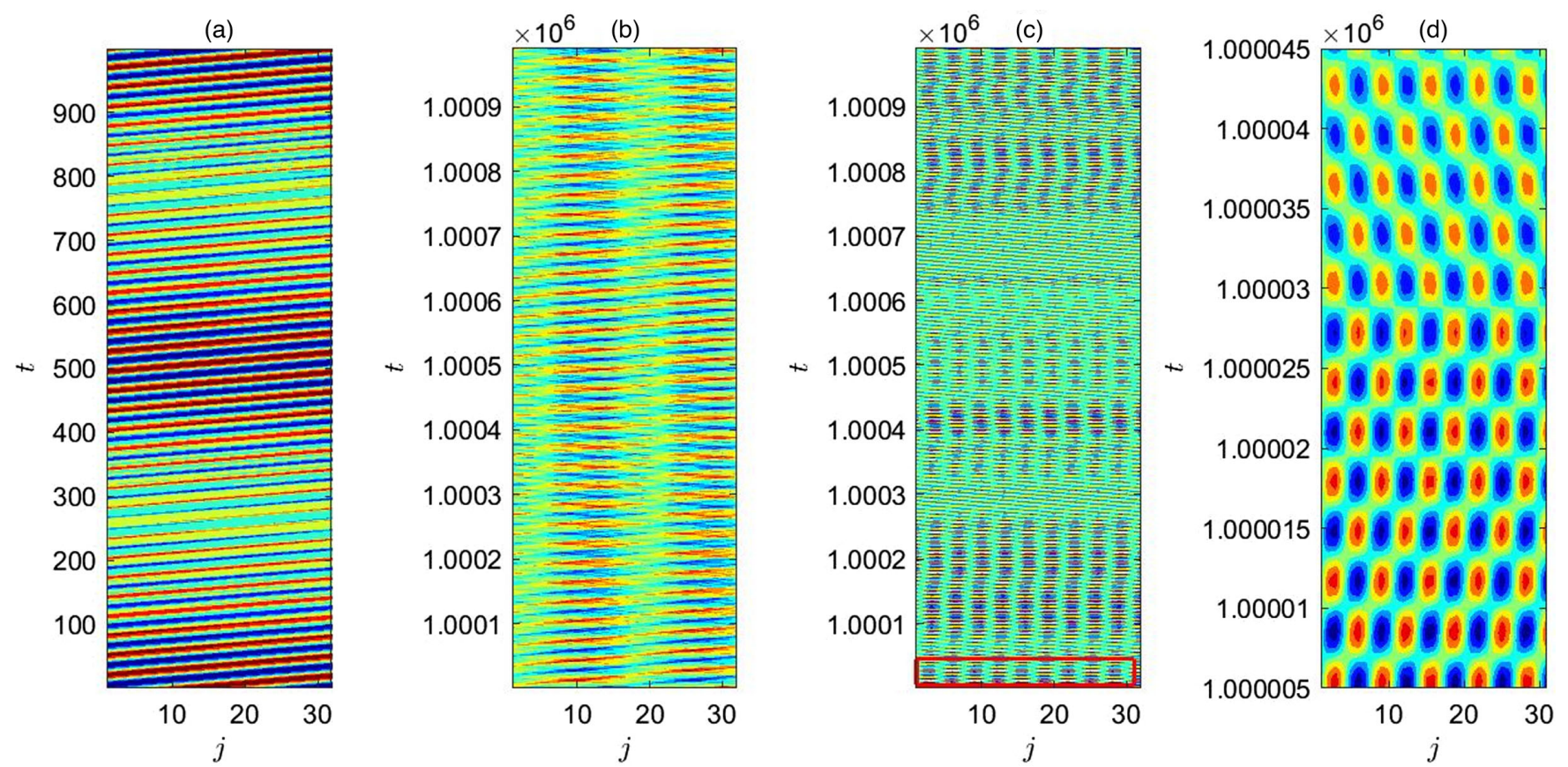

FIG. 9. Color map of the displacement $q_{j}(t)$ for $\epsilon=0.02$ : (a) initial traveling wave, $0<t<1000$, (b) standing wave structure in thermal equilibrium, $10^{6}<t<10^{6}+1000$, (c) $\tilde{q}_{j}(t)$, displacement after removing wave numbers $k=1, \ldots, 4,7, \ldots, N$, (d) closer look at the boxed region in (c).

waves in the selected unfiltered wave numbers, meaning that the coherent structures are not limited to the lowest wave number. Our choice of displaying wave numbers 5,6 is arbitrary; we also verified that similar structures exist over all the wave numbers.
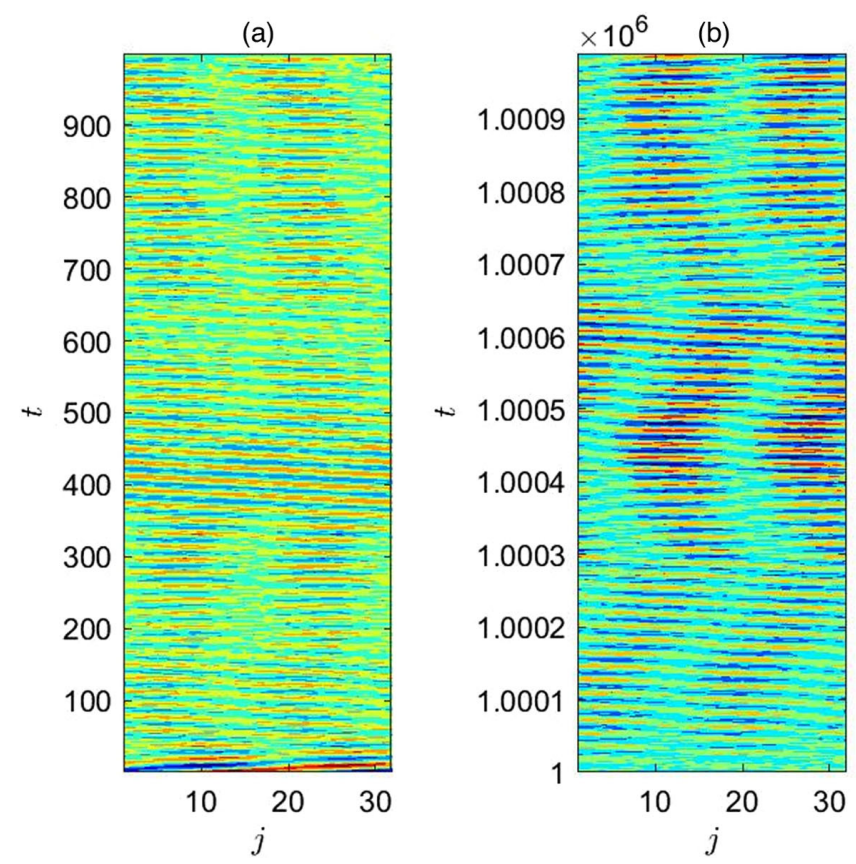

Similarly, in Fig. 10 we obtain similar results for a moderate value of nonlinearity $\epsilon=0.54$ just outside the range of applicability of our theory. We plot the initial time evolution of the displacements in Fig. 10(a), the time evolution of the displacements in thermal equilibrium in

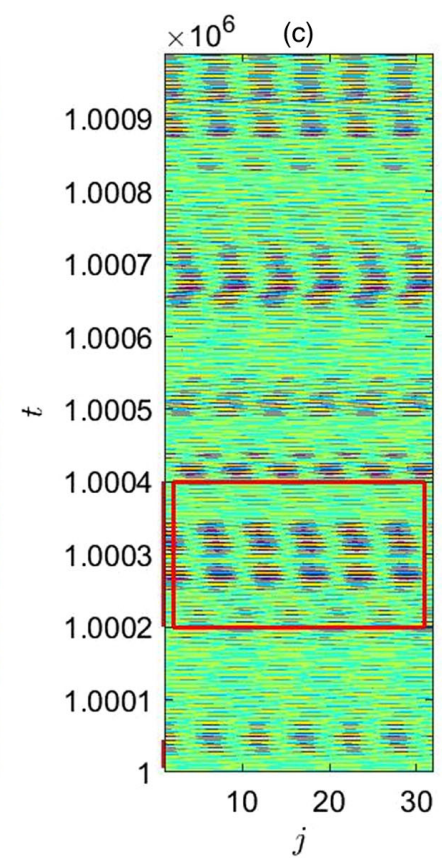

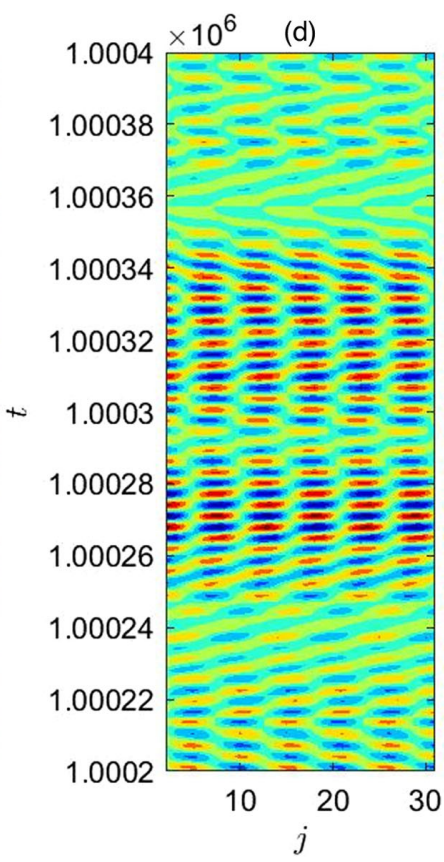

FIG. 10. Color map of the displacement $q_{j}(t)$ for $\epsilon=0.54$ : (a) initial traveling wave, $0<t<1000$, (b) standing wave structure in thermal equilibrium, $10^{6}<t<10^{6}+1000$, (c) $\tilde{q}_{j}(t)$, displacement after removing wave numbers $k=1, \ldots, 3,5, \ldots, N$, (d) closer look at the boxed region in (c). 
Fig. 10(b), and the displacements after applying a spatial frequency filter to emphasize wave number $k=4$ in Fig. 10(c). We note the arbitrary fluctuations between the coherent standing waves and between traveling waves in Figs. 10(b) and 10(c).

\section{CONCLUSION}

In this paper we have given the numerical evidence that anomalous correlators develop spontaneously in a classical system. From a theoretical point of view it is possible to develop a theory for weakly nonlinear dispersive waves that accounts for the presence of such an anomalous correlator. The framework in which the theory has been developed is the wave turbulence one. In such a theory, one usually is interested in the second-order correlator $\left\langle a_{k_{i}} a_{k_{j}}^{*}\right\rangle$, which is strictly related to the wave action spectrum. However, what is clear from numerical simulations of the $\beta$-FPUT system is that also the correlator $\left\langle a_{k_{i}} a_{k_{j}}\right\rangle$ can assume values that are different from zero. This finding has consequences on the standard wave turbulence theory that is based on the Wick's selection rule, i.e., the splitting of higher-order correlators as a sum of products of second-order correlators. Following Refs. [1,15], we have generalized the Wick's rule by including the anomalous correlators. We note that we differ from the case described in the $S$ theory $[9,10]$ in that there, the existence of anomalous correlators was connected with coherent pumping in the system, with the anomalous correlator being a measure of partial coherence for exiting waves. In our observations and predictions, waves with random initial conditions form phase correlations with each other, resulting in an anomalous correlator which is initially zero but then saturates to a nonzero value as it evolves with time.

One of the most striking manifestations of those correlators is the appearance of "ghost excitations," i.e., those characterized by negative frequencies. A formula for the energy content of such excitations as a function of the wave spectrum is obtained - the results compare favorably with numerical simulations for the weakly nonlinear regime. Moreover, we have shown that the spontaneous emergence of the anomalous correlator is strongly connected with the formation of nonlinear standing waves; indeed, the presence of those waves implies a strong correlation between the phases of positive and negative wave numbers.

Our approach paves a new road to investigate dispersive nonlinear systems by taking into account not only amplitudes of the waves, as in traditional wave turbulence, but also the phases of the waves. We conjecture that the anomalous correlators play an important role in the theory of extreme events, such as rogue waves, which form via a mechanism related to phase locking between different wave numbers [29]. Phase locking also leads to the existence of solitons in nonlinear media.

As was discussed in Sec. I, anomalous phase correlations have been observed to play a role in causing shifts of wave action from turbulence and condensate in the nonlinear Schrödinger equation [17]. Our approach of extending wave turbulence theory to include the anomalous correlator could be generalized to address the role these correlations play in the statistical properties of the nonlinear Schrödinger equation and other integrable systems. On a similar note, recurrences in a nonlinear Schrödinger-like model were shown to be directly related to the formation of anomalous phase correlations [20]; further investigating the potential ties between FPUT recurrences and the anomalous correlator is a subject of current work.

Finally, we emphasize that the Hamiltonian we considered is of the same family as the one for surface gravity waves (after removing by a canonical transformation nonresonant three-wave interactions). We predict that also the anomalous correlators will play an important role in the understanding of statistical properties of ocean waves.

\section{ACKNOWLEDGMENTS}

The authors are grateful to Dr. B. Giulinico for discussions. We are grateful to the anonymous referees whose insightful suggestions improved the manuscript considerably. M. O. has been funded by Progetto di Ricerca d'Ateneo CSTO160004, by the "Departments of Excellence 2018/2022" Grant awarded by the Italian Ministry of Education, University and Research (MIUR) (L.232/2016), and by The Simons Foundation, Wave Turbulence. J. Z. and Y. V. L. acknowledge support from NSF OCE Grant No. 1635866. Y. V. L. acknowledges support from ONR Grant No. N00014-17-1-2852.

\section{APPENDIX A: MATRIX ELEMENTS}

Matrix element in Eq. (8).-The matrix elements governing four-wave interactions for the variable $a_{k}(t)$ are

$$
\begin{aligned}
& T_{1234}^{(1)}=-\frac{1}{4} \beta e^{i \pi\left(-k_{1}+k_{2}+k_{3}+k_{4}\right) / N} \prod_{i=1}^{4} \frac{2 \sin \left(\pi k_{i} / N\right)}{\sqrt{\omega_{i}}}, \\
& T_{1234}^{(2)}=-3 T_{1-234}^{(1)}, \quad T_{1234}^{(3)}=3 T_{4231}^{(1)}, \quad T_{1234}^{(4)}=-T_{-1234}^{(1)} .
\end{aligned}
$$

Matrix elements in the canonical transformation, Eq. (22).-The coefficients in Eq. (8) suitable for removing nonresonant terms are given by

$$
\begin{aligned}
& B_{1234}^{(1)}=\frac{T_{1234}^{(1)}}{\omega_{4}+\omega_{3}+\omega_{2}-\omega_{1}}, \\
& B_{1234}^{(3)}=\frac{T_{1234}^{(3)}}{\omega_{4}-\omega_{1}-\omega_{2}-\omega_{3}}, \\
& B_{1234}^{(4)}=\frac{T_{1234}^{(4)}}{-\omega_{1}-\omega_{2}-\omega_{3}-\omega_{4}} .
\end{aligned}
$$




\section{APPENDIX B: EVOLUTION EQUATIONS FOR ANOMALOUS SPECTRUM}

Starting from the transformation in Eq. (22) and the generalized Wick's decomposition in Eq. (21), we obtain

$$
\begin{aligned}
m_{k}^{(a)}(t)= & m_{k}^{(b)}(t)+2\left[n_{k}^{(b)}(t)+n_{-k}^{(b)}(t)\right] \\
& \times \sum_{j} B_{k,-k, j, j}^{(3)} n_{j}^{(b)}(t),
\end{aligned}
$$

where higher-order terms in $m_{k}$ have been neglected. The next step consists in building the evolution equation for $m_{k}^{(b)}(t)$ from Eq. (23). Interestingly, the evolution equation for $m_{k}^{(b)}(t)$ appears as a deterministic dispersive nonhomogeneous wave evolution equation [15],

$$
i \frac{d m_{k}^{(b)}}{d t}=2 \tilde{\omega}_{k} m_{k}^{(b)}+\left[\left(n_{k}^{(b)}+n_{-k}^{(b)}\right) \sum_{j} T_{k-k j-j} m_{j}^{(b)}\right],
$$

with $\tilde{\omega}_{k}=\omega_{k}+2 \sum_{j} T_{k j k j} n_{j}^{(b)}$. Such equations have been derived in the theory of Bose-Einstein condensates and superconductivity.

The equation for the spectrum, see Ref. [15], is given by

$$
\frac{d n_{k}^{(b)}}{d t}=-2 \operatorname{Im}\left[m_{k}^{(b)} \sum T_{k,-k, j,-j} m_{j}^{(b) *}\right] .
$$

From Eqs. (B2) and (B3), after some algebra, it is possible to show that the following interesting relations hold:

$$
\begin{aligned}
& \frac{d\left[\left|m^{(b)}(k)\right|^{2}\right]}{d t}=\frac{d\left[n_{k}^{(b)} n_{-k}^{(b)}\right]}{d t}, \\
& \frac{d\left[n_{k}^{(b)}-n_{-k}^{(b)}\right]}{d t}=0 .
\end{aligned}
$$

If $n_{k}^{(b)}$ has reached energy equipartition such that $n_{k}^{(b)}=n_{-k}^{(b)}=\mathrm{const} / \omega_{k}$, then $\left|m_{k}^{(b)}\right|=n_{k}^{(b)}$; therefore, we expect to observe equipartition also for $\omega_{k}\left|m_{k}^{(b)}\right|$.

We now consider the leading-order solution of Eq. (B2),

$$
m_{k}^{(b)}(t)=m_{k}^{(b)}\left(t_{0}\right) e^{-i 2 \tilde{\omega}_{k} t}+\text { higher-order terms, }
$$

and plug it into Eq. (B1), and assuming that the spectrum $n_{k}$ is in stationary conditions, we get

$$
\begin{aligned}
m_{k}^{(a)}(t)= & m_{k}^{(b)}\left(t_{0}\right) e^{-i 2 \tilde{\omega}_{k} t}+2\left[n_{k}^{(a)}\left(t_{0}\right)+n_{-k}^{(a)}\left(t_{0}\right)\right] \\
& \times \sum_{j} B_{k,-k, j, j}^{(3)} n_{j}^{(a)}\left(t_{0}\right) .
\end{aligned}
$$

Note that we have used the fact that at the leading order $n_{k}^{(b)}\left(t_{0}\right) \simeq n_{k}^{(a)}\left(t_{0}\right)$.

\section{APPENDIX C: DERIVATION OF $(k-\Omega)$ SPECTRUM}

We consider Eq. (22) and take the Fourier transform in time to get

$$
\begin{aligned}
a_{k_{i}, \Omega_{p}}= & b_{k_{i}, \Omega_{p}}+\int \sum_{j, k, l} B_{i j k l}^{(1)} b_{j, \Omega_{q}} b_{k, \Omega_{r}} b_{l, \Omega_{s}} \delta_{i j}^{k l} \delta_{\Omega_{p} \Omega_{p} \Omega_{s}} \Omega_{\Omega_{q}} \\
& +\int \sum_{j, k, l} B_{i j k l}^{(3)} b_{j, \Omega_{q}}^{*} b_{k, \Omega_{r}}^{*} b_{l, \Omega_{s}} \delta_{i j k}^{l} \delta_{\Omega_{p} \Omega_{q}}^{\Omega_{r} \Omega_{s}} d \Omega_{q r s} \\
& +\int \sum_{j, k, l} B_{i j k l}^{(4)} b_{j, \Omega_{q}}^{*} b_{k, \Omega_{r}}^{*} b_{l, \Omega_{s}}^{*} \delta_{i j k l} \delta_{\Omega_{p} \Omega_{q} \Omega_{r} \Omega_{s}} d \Omega_{q r s} .
\end{aligned}
$$

The next step is to build the second-order correlator $\left\langle a\left(k_{i}, \Omega_{l}\right) a\left(k_{j}, \Omega_{m}\right)^{*}\right\rangle$ assuming stationarity.

We use the generalized Wick's decomposition in Eq. (21), i.e., including the anomalous correlators. The leading-order result is contained in Eq. (25).

[1] G. Falkovich, V. S. Lvov, and V. E. Zakharov, Kolmogorov Spectra of Turbulence (Springer, Berlin, 1992).

[2] S. Nazarenko, Wave Turbulence (Springer, New York, 2011), Vol. 825.

[3] J. Benney and A. C. Newell, Random Wave Closure, Stud. Appl. Math. 48, 1 (1969).

[4] A. C. Newell, The Closure Problem in a System of Random Gravity Waves, Rev. Geophys. 6, 1 (1968).

[5] D. J. Benney and P. Saffmann, Nonlinear Interaction of Random Waves in a Dispersive Medium, Proc R. Soc 289, 301 (1966).

[6] B. B. Kadomtsev, Plasma Turbulence (Academic Press, New York, 1965).

[7] Y. Choi, Y. V. Lvov, S. Nazarenko, and B. Pokorni, Anomalous Probability of Large Amplitudes in Wave Turbulence, Phys. Lett. A 339, 361 (2005).

[8] Y. Choi, Y. V. Lvov, and S. Nazarenko, Probability Densities and Preservation of Randomness in Wave Turbulence, Phys. Lett. A 332, 230 (2004).

[9] V. S. Lvov, Wave Turbulence under Parametric Excitation: Applications to Magnets (Springer Science \& Business Media, Berlin, 2012).

[10] V. E. Zakharov, V. S. Lvov, and S. S. Starobinets, Spin-Wave Turbulence beyond the Parametric Excitation Threshold, Phys. Usp. 17, 896 (1975).

[11] P. Janssen, The Interaction of Ocean Waves and Wind (Cambridge University Press, Cambridge, England, 2004), p. 379.

[12] A. Picozzi, J. Garnier, T. Hansson, P. Suret, S. Randoux, G. Millot, and D. N. Christodoulides, Optical Wave Turbulence: Towards a Unified Nonequilibrium Thermodynamic Formulation of Statistical Nonlinear Optics, Phys. Rep. 542, 1 (2014).

[13] D. Proment, S. Nazarenko, and M. Onorato, Sustained Turbulence in the Three-Dimensional Gross-Pitaevskii Model, Physica (Amsterdam) 241D, 304 (2012). 
[14] D. Proment, M. Onorato, L. Vozella, and Y. V. Lvov, A Route to Thermalization in the $\alpha$-Fermi-Pasta-Ulam System, Proc. Natl. Acad. Sci. U.S.A. 112, 4208 (2015).

[15] V. S. Lvov, Wave Turbulence under Parametric Excitations, Applications to Magnets (Springer-Verlag, Berlin, 1994).

[16] J. Bardeen, L. N. Cooper, and J. R. Schrieffer, Microscopic Theory of Superconductivity, Phys. Rev. 106, 162 (1957).

[17] P. Miller, N. Vladimirova, and G. Falkovich, Oscillations in a Turbulence-Condensate System, Phys. Rev. E 87, 065202 (2013).

[18] S. Dyachenko, A. C. Newell, A. Pushkarev, and V.E. Zakharov, Optical Turbulence: Weak Turbulence, Condensates and Collapsing Filaments in the Nonlinear Schrodinger Equation, Physica (Amsterdam) 57D, 96 (1992).

[19] N. Vladimirova, S. Derevyanko, and G. Falkovich, Phase Transitions in Wave Turbulence, Phys. Rev. E 85, 010101 (R) (2012).

[20] M. Guasoni, J. Garnier, B. Rumpf, D. Sugny, J. Fatome, F. Amrani, G. Millot, and A. Picozzi, Incoherent FermiPasta-Ulam Recurrences and Unconstrained Thermalization Mediated by Strong Phase Correlations, Phys. Rev. X 7, 011025 (2017).

[21] E. Fermi, J. Pasta, and S. Ulam, Studies of Nonlinear Problems, I, Los Alamos Scientific Laboratory Report No. LA-1940, 1955.
[22] L. Pistone, M. Onorato, and S. Chibbaro, Thermalization in the Discrete Nonlinear Klein-Gordon Chain in the WaveTurbulence Framework, Europhys. Lett. 121, 44003 (2018).

[23] Y. V. Lvov and M. Onorato, Double Scaling in the Relaxation Time in the $\beta$-Fermi-Pasta-Ulam-Tsingou Model, Phys. Rev. Lett. 120, 144301 (2018).

[24] W. Lee, G. Kovacic, and D. Cai, Generation of Dispersion in Nondispersive Nonlinear Waves in Thermal Equilibrium, Proc. Natl. Acad. Sci. U.S.A. 110, 3237 (2013).

[25] V. Zakharov, Stability of Period Waves of Finite Amplitude on Surface of a Deep Fluid, J. Appl. Mech. Tech. Phys. 9, 190 (1968).

[26] M. D. Bustamante, K. Hutchinson, Y. V. Lvov, and M. Onorato, Exact Discrete Resonances in the Fermi-PastaUlam-Tsingou System, Commun. Nonlinear Sci. Numer. Simul. 73, 437 (2019).

[27] H. Yoshida, Construction of Higher Order Symplectic Integrators, Phys. Lett. A 150, 262 (1990).

[28] V.P. Krasitskii, On Reduced Equations in the Hamiltonian Theory of Weakly Nonlinear Surface Waves, J. Fluid Mech. 272, 1 (1994).

[29] M. Onorato, S. Residori, U. Bortolozzo, A. Montina, and F.T. Arecchi, Rogue Waves and Their Generating Mechanisms in Different Physical Contexts, Phys. Rep. 528, 47 (2013). 